\section{Servier's pipeline in oncology: moving from research to patients}

\begin{abstract}
Patrick Therasse ${ }^{1}$ and Jean-Pierre Abastado*,1 speak to Roshaine Gunawardana, Managing Commissioning Editor: Patrick Therasse is an $\mathrm{MD}, \mathrm{PhD}$ who has focused his career on drug development in oncology. He is currently the Head of Oncology Development for Servier. Before working for Servier, he worked 8 years for GlaxoSmithKline Vaccines as Vice President, Head of Global Clinical Development for immunotherapeutics. He started his career at the European Organization for Research and Treatment of Cancer (EORTC) as a research fellow and then served as Director of the EORTC Research Center for 11 years. He has worked in all fields of oncology with a long track record of publications mainly in the field of breast cancer and methodological research. He is most well known for developing new Response Criteria In Solid Tumours (RECIST), the reference for response assessment in cancer clinical trials since 2000. His areas of expertise encompass Phase I-III drug development with small molecules or active/passive immunotherapy and translational research. Jean-Pierre Abastado is an immuno-oncologist in charge of the R\&D in Oncology at Servier, where he is Director of the Therapeutic Innovation Pole in Oncology. He conducted his professional career between various academic and industrial institutions. He was trained at Ecole Polytechnique (Paris), prepared his PhD on Major Histocompatibility Complex (MHC) at the Pasteur Institute, and made a postdoctoral training at $\mathrm{NIH}$ (Bethesda, MD, USA). He studied T-cell activation in the Immunology department of the Pasteur Institute and at the CNRS. He was Vice President, Chief Scientific Officer of IDM-Pharma, a company specialized in cell therapy against cancer. Before joining Servier, he headed the Laboratory of Tumour Immunology within the Singapore Immunology Network (SIgN) where his team studied the tumor immune microenvironment. He has served on the Editorial board of several journals and in the Scientific Advisory Board of several biotech companies. He has authored more than 120 peer-reviewed publications.
\end{abstract}

First draft submitted: 18 December 2015; Accepted for publication: 21 December 2015; Published online: 14 January 2016

Q How is the field of oncology changing \& how will Servier bring new innovation? Oncology is changing very quickly! There is a massive amount of research being carried out, both in academia and by the pharmaceutical industry. Indeed, there have been dramatic changes in oncology over the last 15 years, with a move from cytotoxic chemotherapy to more personalized targeted cancer therapy, with improved long-term efficacy and better side-effect profiles. For example, 15 years ago, a diagnosis of metastatic melanoma was inevitably a death sentence. Today, survival beyond 5 years is feasible with approved treatments targeting BRAF, CTLA-4, and PD1. The field is moving at an astonishing pace: there were 16 new anticancer agents approved in Europe in 2013, most of which were targeted therapies. The major breakthrough is 'simply' a better understanding of cancer - most notably the process of carcinogenesis, the interaction of cancer cells with the immune system, the process of metastasis and identification of the

'Oncology PIT, IRIS, 50 Rue Carnot, 92284, Suresnes, France

*Author for correspondence: jean-pierre.abastado@servier.com

\section{KEYWORDS}

- hematology $\bullet$ research pipeline

- Servier • solid tumors 
so-called hallmarks of cancer. This has enabled development of new agents that inhibit the very metabolic pathways that underlie cancer cell division and proliferation. In particular, many biotech companies are coming up with new ideas and new products, and so we are moving quickly from a field where most therapeutic options were cytotoxic to a situation that now integrates targeted therapies, personalized medicine and immunotherapy. This means that, in addition to these massive increases in the number of therapeutic options, we can also better characterize patients who are most likely to benefit from a specific treatment.

Servier is expected to play an important role in this process, not only by bringing innovation from our research program, but also due to our initiative to set up synergistic partnerships with academic institutions, biotechnology and pharmaceutical companies. Current research activities are expected to lead to a diversified pipeline of innovative products targeting multiple and complementary cancer hallmarks over the next 10 years. Servier's activity in research and development is currently exploring three main axes: tyrosine kinase receptor inhibition, induction of apoptosis and immunotherapy. Moving closer to the cancer patient, Servier currently has two cytotoxic agents available commercially Muphoran ${ }^{\circledR}$ (fotemustine) and Pixuvri ${ }^{\circledR}$ (pixantrone), and another expected in 2016, Lonsurf $^{\circledast}$ (trifluridine + tipiracil hydrochloride).

Q How important is the role of partnership in research in oncology?

If you walk around the exhibition halls of the major oncology conferences, you can see how many of the smaller companies are coming up with new ideas and new products. Indeed, this is becoming an increasingly important component of drug discovery research in oncology. These partnerships combine the innovation and skills of larger and smaller companies to transform new ideas into products for the patient; it can also accelerate clinical development. To this end, Servier has reached out to a number of institutions, and biotechnology and pharmaceutical partners with recognized track records in oncology. Moreover, we should not forget that good research is necessarily based on fruitful collaboration with academia, and so Servier has also developed scientific collaborations with some of the most prestigious research institutes in oncology, for example, the Curie and Gustave Roussy Institutes in Paris, the Shanghai Institute of Materia Medica and the Singapore Immunology Network in Asia and the Walter and Eliza Hall Institute in Australia. The aim is to maximize the efficiency of our discovery programs.

\section{Q How many cancer products is Servier currently developing \& can we expect any products to become available in 2016?}

We have about 11 products either in 'development' or 'very close to development'. At the moment, exactly nine of the products are in clinical trials and two are expected to begin clinical trials in 2016 or early 2017. For Servier, the most exciting new development for 2016 is Lonsurf, which obtained approval for the treatment of refractory metastatic colorectal cancer from the US FDA in October 2015. Servier signed an exclusive licensing agreement for Lonsurf with the Japanese company Taiho Pharmaceutical Co., Ltd, in June 2015. The direct result of this brand new partnership is that we are highly confident that Servier will be able to launch Lonsurf in Europe in 2016.

Lonsurf is a novel oral combination of trifluridine and tipiracil hydrochloride. Preclinical research suggested that the incorporation of trifluridine into DNA has an antitumor effect, while the tipiracil hydrochloride inhibits an enzyme that degrades trifluridine, thereby maintaining blood concentrations of trifluridine. The results of RECOURSE, a large-scale Phase III trial of Lonsurf in 800 patients with refractory metastatic colorectal cancer, were recently reported in the New England Journal of Medicine (14, 1909-1919 [2015]). Treatment with Lonsurf was associated with a significant improvement in overall survival (7.1 vs 5.3 months; $p<0.001$ ), as well as a longer time to a deterioration in performance status ( 5.7 vs 4 months; $\mathrm{p}<0.001$ ). These results were obtained against a good tolerability profile: the most common toxicities were related to bone morrow suppression and were manageable. Refractory or relapsed metastatic colorectal cancer can be notoriously difficult to treat due to the onset of drug resistance, and the only option left for some patients is to continue with palliative chemotherapy. This is why we are all so excited about the arrival of Lonsurf, as it prolongs survival in heavily pretreated patients with metastatic colorectal cancer and also appears to maintain a good performance status, which is so important for cancer patients and their families alike. 
Q Could you tell us more about pixantrone? Pixantrone (Pixuvri ${ }^{\circledR}$ ) is a very interesting molecule. It currently has a conditional marketing authorization in the EU as a monotherapy for patients with multiply relapsed or refractory aggressive non-Hodgkin B-cell lymphoma. It was originally developed by the US-based biotech company CTI BioPharma, with whom we have been in close partnership since 2014 . Pixantrone has had quite a long development and we are only now starting to understand how it works - and how it works a little differently from what we were expecting. It was initially developed as a kind of anthracycline with decreased cardiotoxicity, but we are discovering that it is actually quite different from the anthracyclines! Pixantrone is a weak topoisomerase II inhibitor, but forms stable DNA adducts, thereby preventing DNA replication and transcription. It appears to be efficacious in anthracycline pretreated patients, without further deteriorating cardiac function in most cases.

The pivotal Phase III results on pixantrone were published in 2012 in the Lancet Oncology (13, 696-706 [2012]). This multicenter, openlabel, randomized trial included 140 patients with aggressive non-Hodgkin lymphoma. They were randomly allocated to receive either pixantrone or a comparator, which was simply the physician's choice of the best available treatment for the patient at that stage of the disease. The results showed a significantly higher complete response rate in pixantrone-treated patients by the end of the treatment period (20.0 vs 5.7\%; $\mathrm{p}=0.021)$. The overall response rate was also better with pixantrone (37.1 vs $14.3 \% ; \mathrm{p}=0.003$ ) and there was a longer progression-free survival. Even better, these results were obtained against an acceptable safety profile, with all toxicities being manageable. These initial results were considered sufficiently promising for the European regulatory authorities to grant a conditional approval for pixantrone, which is excellent news for relapsed or refractory patients who can be very difficult to treat at this stage of the disease. The confirmation of the European authorization will come later with the results of an ongoing CTI BioPharma and Servier collaborative Phase III trial in North American and European patients with aggressive nonHodgkin lymphoma comparing pixantrone and gemcitabine in combination with rituximab. We believe that pixantrone will certainly have a central role to play in the field of management of non-Hodgkin lymphoma patients relapsing after anthracycline, in monotherapy, but also as part of a combination treatment strategy. The hope is ultimately to elicit a response that is sufficiently strong for mobilization of cells for autologous stem cell transplantation and long-term remission.

Q One of Servier's main axes of research in oncology is induction of apoptosis. Can you expand on this?

Evasion to apoptosis (programmed cell death) is one of the hallmarks of cancer; it is also a common mechanism of resistance to drug treatment. This is historically a field in which Servier oncology research has placed a lot of effort. We are expecting much potential for the development of products in the field of apoptosis, with some first-in-class agents and others predicted to represent a whole new family of products.

The BCL-2 family members are known to be crucial inhibitors of apoptosis, acting via the sequestration and inhibition of BAX and BAD, which would normally induce the apoptotic process. Inhibition of the BCL-2 family members would therefore be expected to restore the apoptotic competence of cancer cells. However, because apoptosis is a natural physiological process, it can be very difficult to design molecules specific for BAX, BAD and the BCL-2 proteins without affecting other pathways. Indeed, previous research in this field has only produced agents that blocked several members of the protein family leading to far too many side effects for clinical use. The discovery of specific inhibitors of the main members of the BCL-2 family of proteins was the aim of a close partnership between Servier and Vernalis that was set up in 2007 and extended in 2012. The first agent from this collaboration, S55746, is now in clinical development. This was very quickly picked up by one of our new partners! In May 2014, Novartis signed a partnership with Servier for the development and commercialization of S55746. You can understand why Servier is so convinced that apoptosis will become a very interesting therapeutic option in the next 5 years!

\section{Q Could you also provide more details on} your research on tyrosine kinase inhibitors? We have a few tyrosine kinase inhibitors in the Servier pipeline. The first one is a product we are developing together with Clovis Oncology. It is called lucitanib and at the moment it is being 
developed in the field of breast cancer and in advanced lung cancer. Lucitanib is an agent that targets multiple pathways, including the FGF receptors (FGFR) 1, 2 and 3, the VEGF receptors (VEGFR) 1 and 2 and the PDGF receptor (PDGFR) alpha and beta. There is preclinical and clinical evidence that lucitanib inhibits tumor angiogenesis, and the results of the first-inhuman Phase I/IIa trial were published in 2014 in the Annals of Oncology (25, 2244-2251 [2014]). This open-label study in 76 relapsed or refractory patients with solid tumors in breast, colon, thyroid or lung reported rather spectacular results on tumor regression associated with the presence of necrosis, which is a hallmark of oxygen starvation. The results are currently undergoing further exploration in Phase II clinical trials.

We have two other promising tyrosine kinase inhibitors. One of these is S49076, which comes directly from Servier research itself and is a product being developed to attempt to reverse resistance to some existing targeted and chemotherapeutic agents. $S 49076$ is an oral inhibitor of the HGF receptor cMET, the GAS6 receptor AXL, as well as FGFR. Insofar as these are the most commonly upregulated tyrosine kinase receptors in relapsed patients then we can be fairly optimistic that this agent may address the important unmet need of drug resistance. We are now testing this in the Phase I/II setting in patients with glioblastoma and non-small-cell lung cancer.

The last tyrosine kinase inhibitor $\$ 81694$ is very new - the first patient has recently been treated! It is a cell-cycle MPS1 checkpoint inhibitor, and it is possibly a first in class. To explain, MSP1 is highly expressed in fast-dividing cells, including many cancer cells; MSP1 inhibitors accelerate cell division representing a novel way of destroying cancer cells. $\$ 81694$ was initially developed by Nerviano Medical Science from whom Servier acquired an exclusive license in 2014. We are developing it in both solid and liquid tumors.

\section{Q How is immunology set to revolutionize the treatment of cancer?}

Actually, immunology is already revolutionizing the field of cancer! This has been a long story with 20 years of failures. But now, there is no doubt in the mind of any oncologist that it is a game changer - and is likely to be an even bigger game changer in the future, as we understand more about how the immune system works, which components of the immune system to target, and how these various components are activated. There is a complex interaction between cancer cells and mesenchymal cells in the tumor stroma. In solid tumors, the tumor stroma contains the activated fibroblasts, endothelial cells and immune leukocytes that are essential for tumor growth and metastasis. This includes a whole range of immune cells whose characteristics have proved to predict survival. It is logical to imagine that targeting the interactions between tumors and immune cells would yield new therapies in the fight against cancer - and there have been some successes. The US FDA has recently authorized novel immunotherapies, such as sipuleucel-T in advanced prostate cancer and ipilimumab and nivolumab in unresectable or metastatic melanoma. There have also been impressive results with the use of genetically modified autologous $T$ lymphocytes in patients with acute lymphoblastic and chronic lymphocytic leukemia. We would like to regard immunotherapy as the future fourth cornerstone of cancer therapy, alongside surgery, chemotherapy and radiotherapy.

\section{Q Can you tell us more about Servier's} participation in cancer research in engineered antibodies \& personalized medicine?

We have a few products in the field of immunotherapy. We are currently working with MacroGenics, Inc. to develop engineered antibodies, all of which are first in class. These are what we call Dual-Affinity Re-Targeting, or DART $^{\circledR}$, molecules, in other words, bispecific antibody-like proteins that are designed to target two different antigens. One approach we are pursuing has one target on the tumor cell and one target for the immune $\mathrm{T}$ cells to try to activate the immune system exactly where the cancer cell is - it is very focused! Let us give a concrete example: one of our most advanced DART molecules recognizes both the CD123 on acute myeloid leukemia and myelodysplastic syndrome cells and the CD3 on T lymphocytes. Via this dual recognition, the DART molecule helps form conjugates between cancer cells and lymphocytes, ultimately leading to $\mathrm{T}$-cell receptor aggregation and lymphocyte activation. The overall goal is tumor cell lysis! The real beauty of this is that we can elicit a strong immune reaction against tumor cells via any lymphocyte, independently of its origin. This concept of bispecific targeting has already been demonstrated with blinatumomab in hematology. However, with MacroGenics, we are developing one of these DART molecules 
and have options to develop others for different indications including solid tumors.

Q Is there hope for an 'off-the-shelf' solution with Servier's research in cell therapy?

Yes, there is hope, absolutely! This is something which we are co-developing with a French biotech company called Cellectis. The underlying concept is very appealing to all oncologists. The idea is to use autologous $T$ cells to express chimeric antigen receptors (CARs) that recognize the CD19 antigen, an antigen expressed by leukemia cells, and therefore have antitumor activity. At present, producing autologous CAR-expressing $\mathrm{T}$ cells, so-called CARTs, is a long and tedious process, which has to be repeated for each individual patient separately. The patient's own $T$ cells are collected, purified and then genetically modified to express the tumor-specific CARs. After further steps of purification and amplification, the CART cells are transferred back to the patient. It is laborious, but we now have quite good evidence that CART cell therapy could help a substantial number of patients with hematological malignancies, such as advanced chronic lymphocytic leukemia or acute lymphoblastic leukemia, and it is logical to expect that there will also be future applications in solid tumors. One of the big questions is whether we can expand this beyond being a perpatient approach. The route to this may be to use an allogeneic approach with material provided by healthy donors and re-engineered to support multiple patients with an off-the-shelf solution. In some instances, this might even be the only solution, as patients themselves are unable to provide sufficient substance cells to generate an autologous product.

This is the goal of the partnership between Servier and Cellectis for the development of UCART19. There are a number of challenges to overcome. One is to prevent any graft-versushost disease, by inactivating the endogenous TCR- $\alpha$ gene expressed by the starting T lymphocytes. This appears to be feasible with the Cellectis proprietary technology of TALEN meganucleases. Second, the T cells have to be rendered resistant to destruction by drugs used to eliminate tumor cells. Third, the technique will also require conditioning of the patients so that there is enough space for lymphocyte to expand and persist for some time. Clearly, these are substantial hurdles to overcome, but we believe that the UCART technology could also be a real game changer if a single preparation of modified lymphocytes (UCART19 cells) could provide treatment for several - if not hundreds - of patients. Ongoing research will also explore whether this therapy could be applied in other types of cancer, including solid tumors. This is our hope for an 'off-the-shelf' solution for patients. And it is very, very promising.

\section{Q How will these developments affect}

clinicians \& their patients \& when can we start to see the benefits?

We have two products currently in launch phase, or very close to launch, Pixuvri and Lonsurf. Patients can start to benefit from these agents immediately or in the very near future. As regards to the Servier research pipeline, we have seen that some of our products are very innovative and could be real game changers. We will know more as soon as we start clinical trials assessing the efficacy of our products. This means that the impact for patients could be seen very quickly, as was the case for autologous UCART19 cells. On the other hand, it may take a bit more time if we have to go through large Phase II and III clinical trials. Our current estimation is that we could start seeing the next new products coming out of the Servier research pipeline within a range of 2-3 years if one or more products are exceptionally active.

\section{Q Do you believe that combination therapy} is the ultimate way forward in oncology?

Combination therapy is certainly one of the options we can take to develop a product. Combination therapy is being increasingly used, particularly in combining investigational products - and that is the right thing to do. On the other hand, this should not be the ultimate goal. Monotherapy will and should have a place in the management of cancer - there is no discussion on that - but we see that we can leverage some of these products by combining them with good scientific rationale. Sometimes, we can be very surprised about the synergy we find between two agents! So yes, combination is much more on the radar screen earlier on in development than it was before, but it is not the only way forward in oncology!

\section{Q Where do you see Servier's research in} oncology in 10 years' time?

Certainly, we see Servier as being a relevant and important partner in oncology in the next decade. We have a portfolio with innovative approaches which could reveal early on as breakthrough therapies, notably in the field of immunotherapy and apoptosis, which are our 
two main axes of research. In this field, there is certainly a bright future for Servier.

Q Finally, do you believe a cure for cancer is a possibility?

I believe that a return to health after cancer is possible for some patients - the number of which will increase in the years to come. On the other hand, whether we can really call this a 'cure for cancer' is debatable. If you ask the medical oncologists, they will tell you that they do not like to talk about cure for cancer. They tend to believe that they can transform the disease into a benign form, in other words, take patients into long-term remission. But nobody can really commit to this being a cure. However, some patients will certainly not have their disease coming back at all.

\section{Q Do you have any closing remarks for our} readers?

As a summary, Servier currently has an exciting pipeline in oncology with an array of new products, many of which have the potential to be game changers. The examples of the collaborations between Servier and our various industrial and academic partners show just how important effective partnership is to accelerate the delivery of new drugs to the patients who need them and we believe that there is plenty of room for more collaboration. The success of these partnerships appears to be independent of whether the partners are big pharma or small biotech: it all depends on effective collaboration, which is one of Servier's great strengths. Everyone has a valuable role to play in the fight against cancer.

\section{Disclaimer}

The opinions expressed in this interview are those of the interviewees and do not necessarily reflect the views of Future Medicine Ltd.

Financial \& competing interests disclosure $P$ Therasse and J-P Abastado are employees of Servier. $P$ Therasse and J-P have no other relevant affliations or financial involvement with any organization or entity with a financial interest in or financial conflict with the subject matter or materials discussed in the manuscript apart from those disclosed.

P Therasse and J-P Abastado thank Sarah Novack, who provided medical writing assistance on behalf of Servier, France. 